\title{
Entre cognition et émotion : les potentialités de l'art dans l'éducation thérapeutique
}

\section{Potentialities of Art in Therapeutic Education: Between Cognition and Emotion}

\author{
Alessandra PELLECCHIA ${ }^{1}$, Rémi GAGNAYRE
}

\begin{abstract}
Résumé L'expérience artistique, du créateur ou du récepteur de l'ouvre d'art, en provoquant des altérations de l'état émotionnel, fait appel à des fonctions cognitives. Problématique : Cette étude vise à éprouver l'expérience esthétique, en particulier la relation avec une ouvre d'art, comme un support psycho-émotionnel possible dans l'expérience de la maladie. Hypothèse théorique : Des approches spécifiques de l'art, intégrées dans un programme d'éducation thérapeutique, peuvent favoriser l'apprentissage des compétences d'autosoins et d'autosurveillance, en contribuant aux processus de : réhabilitation du sentiment d'identité et revalorisation de l'image de soi; acceptation de la maladie et compréhension du traitement; appréhension des représentations sociales de la maladie ; gestion des émotions. Projet-perspectives : La validation de l'hypothèse théorique ci-dessus formulée nécessite la mise en place d'un programme expérimental d'éducation thérapeutique intégrant l'expérience esthétique comme support pédagogique. Evaluation prévue : Questionnaires et entretiens pour savoir comment et combien le comportement des participants a évolué à la suite du programme; savoir la place que les participants attribuent aux expériences esthétiques dans la mobilisation des capacités relationnelles et émotionnelles visées par le programme. Conclusion : L'expérience esthétique, intégrée dans un programme d'éducation thérapeutique, pourrait favoriser le développement de deux groupes de capacités de la part du patient : 1) pour la gestion des émotions; 2) pour la prise de conscience d'un décalage entre les représentations personnelles et celles d'autrui ainsi quentre les représentations et la réalité.
\end{abstract}

Mots clés Emotions ; représentations ; apprentissage ; art ; éducation thérapeutique des patients.

Abstract Art, in its capacity to trigger emotional reactions and to stimulate our cognitive functions, appears to be particularly well adapted to convey educational messages. Problematic: To assess an aesthetic experiment by means of an association of a work of art (as a physcho-emotional support) with an experienced disease. Theoretical assumption: When integrated in therapeutic educational programs, specific approaches of art may help people to learn how to cure and supervise themselves by contributing in the process of: 1) the rehabilitation of the patient's identity, 2) the acceptance of one's illness, 3) the perception and representation of the illness itself and its treatment and 4) the patient's understanding of various social perceptions regarding his illness. Project: The validation of the theoretical hypothesis mentioned above requires the implementation of an experimental program of therapeutic education, which integrates the aesthetic experiment as a teaching support. Anticipated evaluation: Questionnaires and interviews will be given to in order: 1) to know how and how much the behavior of participants evolved subsequently to the program and 2) to know how participants ascribed the aesthetic experiment in regards of the mobilization of relational and emotional capacities aimed in the program. Conclusion: If integrated in a therapeutic education program, the aesthetic experiment could support the development of two different capacities: 1) managing emotions and 2) becoming aware of the shift existing between personal representations and those of others as well as the gap between representations and reality.

Key words Emotions; representations; learning; art; therapeutic patient education.

Pédagogie Médicale 2006;7:101-9

1- Laboratoire de Pédagogie de la Santé - EA 3412 - Université Paris 13 - UFR SMBH Léonard de Vinci.

Correspondance : Rémi Gagnayre - Laboratoire de Pédagogie de la Santé - EA 3412 - Université Paris 13 - UFR SMBH Léonard de Vinci 74 rue Marcel Cachin - 93017 Bobigny Cedex - France - Téléphone : +33 (0)1 48387645 - mailto:r.gagnayre@smbh.univ-paris13.fr 


\section{Introduction}

La culture, comme les sciences, dans les sociétés occidentales a été traditionnellement et profondément conditionnée par une vision opposant la sphère de la raison, de la cognition et de l'esprit à la sphère de l'instinct, de l'émotion et du corps. L'histoire de la philosophie pérennise pour des siècles cette opposition.

Entre les différentes conceptions philosophiques de l'art, nous retrouvons une conception "esthétique " et une conception « cognitive ». La première, dite aussi « anticognitive " pour souligner l'incompatibilité entre les deux, affirme que la création artistique est un mystère, celui du génie. Dans l'expérience esthétique, l'appréhension de l'œuvre d'art serait de l'ordre du plaisir immédiat et non de la compréhension intellectuelle. La conception cognitive, à l'opposé, affirme que la création artistique suppose l'inventivité et l'originalité, mais dans la même mesure que la création scientifique et technique. Relativement à l'expérience esthétique, cette deuxième conception soutient que la compréhension et l'appréciation d'une œuvre d'art font appel à des fonctions strictement intellectuelles. Cette opposition a été finalement mise en cause par la théorie esthétique proposée par Nelson Goodman, «théorie qui offre un cadre conceptuel permettant d'intégrer de manière cohérente les composantes perceptuelles et symboliques, empiriques et formelles, émotionnelles et intellectuelles de l'expérience artistique $»^{1}$.

Une autre conception de l'art, la conception "éthique ", se place sur un plan tout à fait différent mais qui nous parait cohérent avec la logique de notre proposition. Cette conception affirme que l'expérience esthétique permet d'appréhender certaines valeurs morales qui ne pourraient être autrement formulées de manière satisfaisante par le discours. La conception éthique met en avant le caractère profondément humain de l'art. L'expérience esthétique vécue par le récepteur ou le créateur est liée à la vie morale et aux relations sociales. L'art donne à vivre une expérience humaine ${ }^{2}$.

L'expérience esthétique est nécessairement la création d'un sens entre sujet et objet. Pour l'artiste ce sens prend forme à l'aide de ses instruments et de son intention artistique. Il est créateur au premier sens du terme. Il donne vie à la matière : il compose, il peint, il sculpte. Dans l'expérience esthétique, le récepteur de l'œuvre d'art n'échappe pas à cette nécessité de création de sens. Il est en pleine jouissance de l'œuvre, se l'approprie et finit par la recréer à sa convenance. Le récepteur réalise une création imaginaire grâce à son interprétation subjective de l'œuvre d'art. L'étude décrite dans cet article se réfere à cette expérience particulière du récepteur.
A la suite des perspectives ouvertes par les récentes recherches d'inspiration cognitive, l'idée qu'une expérience humaine s'inscrit dans une dimension exclusivement émotive ou cognitive devient inacceptable. L'expérience artistique, du créateur ou du récepteur, en provoquant des altérations de l'état émotionnel, fait appel à des fonctions cognitives : reconnaissance des formes, compréhension et production du langage, acquisition de connaissances, conduite du raisonnement. La dimension émotionnelle et la dimension cognitive ne peuvent qu'être co-intéressées en tant qu'aspects d'un phénomène unique.

Cet antagonisme entre dimension cognitive et dimension émotive intéresse la pédagogie. Les professionnels de l'éducation ont traditionnellement considéré les émotions comme un obstacle dans les processus d'apprentissage. Au cours du siècle dernier, la dimension émotionnelle a pris de plus en plus de place dans les théories et dans les pratiques pédagogiques. Cette dimension, toutefois, a été considérée plutôt comme contextuelle ou motivationnelle et non pas comme partie intégrante du processus d'apprentissage .

La reconnaissance de l'intelligence émotionnelle a produit des changements importants. La notion d'intelligence émotionnelle comprend : connaissance et maitrise de ses émotions, automotivation, perception des émotions d'autrui et maitrise des relations humaines. Cette forme d'intelligence est aujourd'hui reconnue comme facteur significatif de réussite scolaire et professionnelle. Goleman en particulier affirme que les habiletés émotionnelles et les habiletés cognitives se rejoignent dans l'état de "fluidité ", summum de l'intelligence émotionnelle et situation idéale pour l'apprentissage et la performance ${ }^{4}$. "Dans cet état de grâce, en effet, l'individu se laisserait porter par le plaisir de l'expérience, perdrait toute notion d'espace et de temps comme de son existence propre, et dégagerait toute son énergie au service de la tâche entreprise. Son attention serait à la fois détendue et très focalisée et son état affectif s'établirait dans l'harmonie, en deçà de l'inquiétude qui inhibe et au-delà de l'ennui qui démobilise. Même les travaux les plus difficiles lui sembleraient reposants ou réparateurs plutôt qu'éprouvants. Ainsi saurait-il canaliser ses émotions au service de son intérêt, retarder la satisfaction de ses désirs et contrôler son humeur pour faciliter sa pensée au lieu de l'entraver, persévérer malgré ses échecs et développer le maximum de son potentiel intellectuel " . Les aspects affectifs et émotionnels doivent être pris en compte pour favoriser les processus d'apprentissage et supporter la motivation à apprendre. Premièrement, cette prise en compte permettra de développer les attitudes et les valeurs favorables aux apprentissages ${ }^{6}$, de dépasser les résistances à l'apprentissage. Deuxièmement, grâce à la 
recherche dans le domaine de la psychologie cognitive et de la neuropsychologie, nous savons que l'apprenant qui apprécie la conduite et les contenus de son apprentissage pourra maintenir son intérêt et faire appel à ses habiletés intellectuelles supérieures et métacognitives. La recherche de solutions pédagogiques capables de stimuler le plaisir à apprendre et de favoriser l'atteinte de l'état de fluidité de la part de l'apprenant est donc essentielle.

La notion d'apprentissage, en tant que " processus d'acquisition d'un savoir, d'un savoir-faire, d'un savoir-être, produisant un changement de comportement " ${ }^{7}$, nous amène à réfléchir sur le rôle des représentations. Dans le processus d'apprentissage, des informations ou bien des expériences nouvelles sont intégrées dans le système des connaissances, des compétences ou des capacités antérieures, en produisant un effet sur les représentations. Laccroissement des savoirs se traduit par un renforcement, un questionnement ou une modification des représentations $^{8}$. En d'autres termes, les représentations sont, à la fois, un facteur d'apprentissage et un objectif de l'apprentissage.

Afin d'éclaircir le paradigme apprentissage - connaissance - représentations, nous rappelons que " connaître une chose c'est se l'assimiler, se la rendre intérieure, la faire sienne, et ainsi se la rendre présente [...] tout en lui laissant son statut de réalité extérieure. [...] La connaissance apparaît ainsi comme une sorte de redoublement du monde...». La connaissance est faite de représentations. Nous pouvons identifier trois niveaux de connaissance ${ }^{9}$ : 1) perception sensible : connaissance liée à la présence physique de la chose connue. La représentation est le résultat de la perception par les organes sensoriels (effet sur les terminaisons nerveuses, effet au niveau du système nerveux central, image mentale associée).

2) Connaissance imaginative : représentation d'un objet qui n'est pas physiquement présent, grâce à la reproduction d'un objet antérieurement perçu ou grâce à l'évocation d'un objet nouveau construit à partir de fragments de la réalité perçue (image évocatrice).

3) Connaissance abstraite : l'objet est saisi non dans sa réalité concrète, mais dans un de ses aspects particuliers qui peut se trouver réalisé en d'autres objets (concept).

Le mécanisme de la représentation se réalise et se spécifie selon ces trois niveaux de la connaissance : sensibilité, imagination, intellect.

Dans le processus d'apprentissage, l'acquisition de nouvelles informations ou expériences se déroule sur des niveaux conséquents : mise en présence du sujet par rapport aux contenus de l'apprentissage, intériorisation de ses contenus, (re)productions de ces contenus dans l'espace de la conscience. Les représentations servent à interpréter les objets de connaissance, à s'expliquer les expériences vécues, à donner un sens aux choses du monde avec lesquelles on rentre en relation ${ }^{10}$.

Dans la conception de programmes pédagogiques, il est indispensable de prendre en compte les représentations des destinataires comme un facteur essentiel de l'apprentissage. Les programmes pédagogiques doivent identifier et comprendre les représentations des destinataires pour proposer des solutions pédagogiques adaptées, faute de quoi ces programmes ne pourront pas être efficaces. En proposant des apprentissages structurés et ciblés, ils devraient avoir comme objectif intrinsèque la prise de conscience d'un décalage entre ses représentions personnelles et celles d'autrui ainsi qu'entre ses représentations personnelles et la réalité. Le système des représentations et des émotions de la personne en situation d'apprentissage constitue le terrain psycho-affectif dans lequel iront s'intégrer les nouvelles connaissances, compétences et capacités.

\section{Problématique}

La finalité de l'éducation thérapeutique est d'apprendre aux malades chroniques à avoir un rôle actif et responsable dans leur thérapie et dans leur suivi médical. Plus largement, l'éducation thérapeutique a pour but d'éduquer à "bien vivre" tout en étant atteint par une maladie chronique. L'éducation thérapeutique s'est adressée premièrement aux diabétiques, puis à des patients atteints de pathologies chroniques variées, en privilégiant l'asthme et l'obésité. Actuellement, le champ d'action de l'éducation thérapeutique tend à s'élargir :

- sur le plan des maladies concernées par cette discipline. Grâce aux progrès dans les soins médicaux et dans le diagnostic, les patients atteints par certaines maladies auparavant considérées comme mortelles, voient leur espoir de vie s'allonger considérablement. Nous nous trouvons donc confrontés à la problématique des "nouvelles » maladies chroniques ;

- sur le plan de la sphère psycho-affective du patient, soit en tant que facteur de processus d'apprentissage, soit facteur d'influence de la qualité de vie des patients.

L'hypothèse, ici proposée, se place à l'intersection de ces deux courants d'évolution. Premièrement, elle s'adresse aux malades atteints d'un cancer, pathologie qui, dans certaines de ses formes, peut être considérée comme une maladie chronique. Deuxièmement, l'étude ne s'intéresse pas directement au processus d'apprentissage, mais au terrain psycho-émotionnel qui le favorise.

Afin d'observer et de mieux comprendre le contexte psycho-émotionnel des patients, nous avons focalisé notre 
attention sur les processus suivants : perception/représentation de soi et du corps ; perception/représentation de la maladie et du traitement ; influence des représentations sociales de la maladie; gestion des émotions.

Ces processus sont, plus au moins consciemment, mis en place par les patients. La façon de conduire ces processus favorise ou non l'apprentissage des compétences nécessaires à gérer la maladie chronique. Comment aider les patients à bâtir un contexte psycho-émotionnel propice à son apprentissage ? Quel support proposer ?

Cette étude vise à éprouver l'expérience esthétique, en particulier la relation avec une ouvre d'art, comme un support psycho-émotionnel possible dans l'expérience de la maladie.

Lart a le pouvoir d'influencer le regard que nous portons sur le monde. L'œuvre d'art fixe le regard de l'artiste, sa façon de percevoir les choses au moment de la création. Ce regard original, parfois marginal, favorise la conscience de la multiplicité des regards possibles par rapport à une réalité unique mais insaisissable. Le récepteur est confronté à une perception/représentation nouvelle de l'objet de l'œuvre. Cette expérience, de l'ordre de la révélation, favorise et renforce la capacité de regarder autrement le monde entourant, de développer un regard personnel et libéré des schémas conventionnels.

Dans une structure de soins, l'art peut modifier le regard du personnel soignant sur l'établissement, des familles sur le personnel soignant, des artistes sur des questions cruciales pour leur travail, touchant à des points essentiels tels que la vie, la mort, la souffrance ou le corps. L’art peut également modifier la façon dont les patients perçoivent l'hôpital, leur maladie, leur vie.

La personne qui se découvre malade perd ses repères, les espoirs et les valeurs qui avaient caractérisé sa vie jusqu'alors. Elle aurait besoin de réviser son projet de vie mais, souvent, plus que les forces physiques, les forces morales et psychologiques lui manquent. La maladie, en particulier la maladie chronique, est vécue comme une condamnation à ne plus vivre. L'art suggère à la personne malade une autre façon de vivre la maladie et de concevoir les soins. C'est pourquoi le traitement thérapeutique peut se révéler l'occasion d' " ouvrir des portes sur soi-même " ${ }^{11}$.

Ces intuitions sont confortées par certaines connaissances dans le domaine des sciences cognitives selon lesquelles l'émotion et la cognition sont des aspects d'un même phénomène : la perception du monde. La relation au monde déclenche des sensations, voire des émotions, et corrélativement elle provoque une activité cognitive (analyse et sélection des informations, prise de décisions, etc, par rapport aux expériences antérieures). Comment se traduit dans le domaine cognitif la relation avec une œuvre d'art, c'est-à-dire avec un objet qui existe pour transmettre des émotions ? Est-ce que les retombées d'une expérience esthétique peuvent être utiles au bien-être du patient et à la finalité de l'éducation thérapeutique (autosoins et autosurveillance) ? A ce titre, l'expérience esthétique mérite d'être explorée et expérimentée comme vecteur d'un changement dans la façon que le patient a de s'appréhender, de se comporter et de se soigner.

\section{Hypothèse théorique}

L'art, en tant que déclencheur d'expériences émotionnelles et stimulateur de fonctionnalités cognitives, nous semble particulièrement adapté à véhiculer des messages pédagogiques de façon efficace et, en même temps, originale, agréable et captivante. Il semble possible de tester les potentialités de l'art ci-dessus supposées, en particulier auprès de personnes atteintes d'un cancer et nécessitant un traitement de longue durée.

Dans ce contexte pédagogique, les destinataires présentent une situation émotionnelle sensibilisée et marquée par le vécu de leur maladie. La motivation à apprendre comment gérer et traiter leur maladie est en relation étroite avec la qualité du climat thérapeutique.

L'analyse de témoignages de malades (interrogés dans le cadre d'autres expériences ${ }^{12,13}$ ou issues de la littérature ${ }^{14-16}$ ) révèle des aspects assez récurrents dans l'expérience du cancer : une diminution de l'estime de soi accompagnée et renforcée par une dégradation de l'image de soi (celle-ci due à certaines thérapies plutôt qu'à la maladie ellemême); la recherche d'un sens à donner à la maladie (pourquoi moi ?) qui est fondamentale dans le processus d'acceptation de la maladie ; l'influence négative des représentations sociales par rapport à la représentation personnelle de la maladie.

Des expériences esthétiques spécifiques, intégrées dans un programme d'éducation thérapeutique, peuvent favoriser l'apprentissage des compétences d'autosoins et d'autosurveillance, en contribuant aux processus de : réhabilitation du sentiment d'identité et revalorisation de l'image de soi ; acceptation de la maladie et compréhension du traitement ; appréhension des représentations sociales de la maladie ; gestion des émotions.

Lart représente un support pédagogique privilégié parce qu'il donne accès à la sphère des émotions ainsi qu'à la sphère des connaissances et des représentations.

\section{Potentialités de l'art par rapport à la sphère cognitive et aux représentations}

L'œuvre d'art est l'exemple le plus pertinent et efficace du principe de la multiplicité de significations possibles. Ce 
que l'artiste a voulu représenter n'est pas toujours ce que le récepteur de l'œuvre voit ou entend. L'œuvre d'art est filtrée par l'imaginaire de celui qui la reçoit, interprétée à la lumière des expériences personnelles. Son sens change avec son récepteur.

Le malade, grâce à la métaphore de l'œuvre d'art, prend conscience de son pouvoir de se percevoir autrement, de changer son regard sur lui-même, sur sa maladie et sur sa vie. La maladie peut assumer un sens auparavant ignoré et le programme d'éducation thérapeutique peut se révéler une occasion pour ouvrir des portes sur soi-même.

Le cancer, comme le SIDA, est caractérisé par des représentations sociales particulièrement lourdes ${ }^{17}$. Les représentations sociales peuvent rendre la maladie plus dramatique et douloureuse pour qui en est affecté. La personne qui se découvre malade redoute des conséquences physiques (perte des capacités motrices, douleurs), des conséquences psychologiques (diminution des ressources psychologiques, dépression) et des conséquences sociales (perte du rôle social, marginalisation). Entre toutes, les conséquences sociales peuvent être les plus graves et difficiles à affronter. Pour les personnes affectées par ces maladies, il devient donc plus important, et dans le même temps plus difficile, d'apprendre à vivre l'expérience de la maladie sans tomber dans le piège de la représentation de la maladie chronique comme situation de «non vie".

Grâce aux expériences esthétiques spécialement intégrées dans un programme d'éducation thérapeutique, le malade du cancer peut apprendre à considérer les représentations sociales autrement, en ayant pris conscience de leur relativité et précarité. Le patient devient capable de construire de façon plus créative et autonome la représentation de soi et de son expérience.Par ailleurs, le fait de se sentir autorisé à recevoir une ouvre d'art, de faire partie d'un contexte intellectuellement motivant, de se voir appelé à s'exprimer autour d'un sujet privilégié, comme l'art peut l'être, améliore l'image que le patient a de lui-même.

\section{Potentialités de l'art par rapport à la sphère émotionnelle du patient}

L'œuvre d'art est émotion ${ }^{18}$. Elle matérialise celles de son créateur et elle suscite des émotions auprès de ses récepteurs.

Le patient, grâce à la découverte d'œuvres d'art, peut apprendre à gérer ses émotions. D'abord, il apprend à reconnaître avec plus d'acuité ce qui est pour lui source d'émotions positives et ce qui est source d'émotions négatives. Il découvre que l'expérience esthétique est source de sens ou - nous pourrions dire autrement - qu'elle est source de joie. En effet, la joie peut être considérée comme « le pouvoir d'être libre de la tyrannie des émotions négatives ${ }^{19}$.

La rencontre avec une œuvre d'art laisse le temps de réfléchir sur ce qui s'est passé. Le patient, opportunément accompagné et stimulé, parvient à focaliser ses émotions. Il prend une distance par rapport à celles-ci. Cette distanciation constitue le présupposé essentiel pour acquérir une gestion de ses émotions. Le sentiment de pouvoir ne pas se laisser emporter par ses propres émotions renforce la confiance en soi et l'estime de soi.

Dans le cadre d'un programme d'éducation thérapeutique, le patient peut apprendre à reconnaître ses émotions pour arriver à les gérer. Nous nous attendons à ce que le patient développe les capacités nécessaires à :

- prendre conscience de ses émotions (savoir les reconnaître et les nommer) ;

- prendre distance par rapport à ses émotions (changement de perspective : de l'intérieur à l'extérieur) ;

- gérer ses émotions (recherche des moyens pour les canaliser).

Le patient doit apprendre à porter un autre regard sur sa maladie et sur les émotions qu'elle provoque : en regard extérieur. Nous pensons que pour réaliser ce changement de perspective, fondamental pour la gestion des émotions, la rencontre avec une œuvre d'art est un moyen plus pertinent de la création matérielle. Cependant, dans une expérience esthétique l'observateur ne reste pas passif ; au contraire, il réalise une activité de création imaginative représentée par son interprétation de l'œuvre d'art. Grâce à sa lecture (ou relecture), il créera un nouveau sens de l'œuvre. Il devrait donc apprendre à transférer les capacités apprises au regard de l'œuvre d'art à son vécu de maladie. Il peut être opportun de spécifier que les expériences proposées pour valider notre hypothèse théorique, sont qualifiées comme esthétiques puisque cette étude n’envisage pas une production artistique de la part des patients (contrairement aux ateliers de création artistique ou d'artthérapie qui offrent aux malades d'autres formes de soutien). Dans le même esprit, mais sur des bases différentes, le service d'enseignement thérapeutique pour maladies chroniques des Hôpitaux universitaires de Genève a mis en place, depuis 2002, un programme d'Art-thérapie dans les soins (AR-T-S). Des patients atteints de maladies chroniques ont la possibilité d'exprimer leur vécu de la maladie grâce à des démarches d'expression artistique. Le programme consiste en trois ateliers. Dans l'atelier "Ecritoire», les patients expriment leur vécu par l'entremise de l'écriture et de la mise en scène. Le récit est mis en scène par les participants et il est joué par des acteurs professionnels, sous la direction d'un metteur en scène lui aussi professionnel. Cet atelier produit un effet de distanciation du patient par 
rapport à son vécu qui lui permettrait de mieux vivre avec sa maladie.

Grâce à l'expression picturale, l'atelier "Trace " vise un " lâcher-prise de l'intellectuel en faveur des sensations corporelles, visuelles et kinesthésiques ». "Tableau de Vie ", troisième atelier du programme AR-T-S, s'adresse à des personnes âgées, dans le but « d'intégrer leur expérience au projet de réhabilitation $»^{20}$.

Dans notre hypothèse, les patients créent dans la mesure où ils traduisent et interprètent l'œuvre d'art à laquelle ils sont confrontés. L'expérience esthétique est utilisée comme déclencheur d'une réaction émotionnelle.

\section{Projet et perspectives}

La validation de l'hypothèse théorique ci-dessus formulée nécessite la mise en place d'un programme expérimental d'éducation thérapeutique intégrant l'expérience esthétique comme support pédagogique, ainsi qu'un dispositif d'évaluation adapté.

L'expérimentation s'inspire de la méthode de la rechercheaction et se réalise grâce à un cycle de rencontres avec un groupe de patients atteints d'un cancer. L'outil des entretiens nous semble le plus adapté au recueil et à l'analyse d'informations concernant les situations psychologiques et affectives des patients. Pour valider l'hypothèse et aboutir aux objectifs de l'étude, nous allons privilégier les entretiens de groupe avec les participants au programme.

Nous avons prévu quatre rencontres, une par semaine pendant quatre semaines consécutives. La durée de chaque réunion est d'environ trois heures. Chaque réunion se focalise sur un ou plusieurs processus psychoaffectifs grâce à leur mise en relation avec une ouvre d'art (spécialement retenue pendant une phase préalable de l'étude). L'équipe pédagogique est composée par :

- le médiateur, avec des compétences en éducation thérapeutique ;

- l'auteur de l'œuvre d'art proposée, chargé de présenter l'œuvre et de se confronter à ce sujet avec les patients ; - le psychologue, qui pourra intervenir si nécessaire. Chaque réunion se déroulera selon le schéma suivant : présentation de l'œuvre d'art ; réactions/groupe de parole ; tentative de mise en relation entre l'expérience de la maladie et l'expérience esthétique vécue.

Grâce à ces réunions, nous espérons aider les patients à prendre conscience des compétences et des capacités qu'ils devraient mobiliser pour mieux gérer leur maladie et leur vie. L'effort cognitif nécessaire à la mobilisation de ces capacités et compétences est facilité par la charge émotive due à l'expérience esthétique vécue.

La conception et la réalisation d'un programme d'éduca- tion thérapeutique intégrant des dimensions artistiques ne peuvent être assurées, nous semble-t-il, que par une équipe pluridisciplinaire comprenant des compétences pédagogiques, médicales, psychologiques et artistiques. L'implication et la mise en collaboration de spécialités professionnelles diverses sont nécessaires à la réflexion et à la prise de décisions sur les objectifs pédagogiques, sur la façon d'intégrer l'art dans la stratégie pédagogique et sur les outils pédagogiques les plus adaptés. Les compétences médicales et les compétences pédagogiques peuvent bien évidemment être assurées au sein de l'équipe par un éducateur thérapeutique. Dans ce cas, celui-ci doit avoir les compétences propres à la profession d'éducateur thérapeutique, telles qu'elles sont décrites dans le rapport technique du groupe de travail OMS - Europe ${ }^{21}$, ainsi que des compétences spécifiques pour intégrer l'art dans les contenus de programmes d'éducation thérapeutique. On peut signaler aux moins trois groupes de compétences pour les éducateurs :

1) développement du patient : proposer des approches de l'art favorisant une transformation émotionnelle et cognitive du patient propice au développement de la découverte de soi après la maladie, de la compréhension de la maladie et du traitement, de l'acquisition des compétences d'autosoins et d'autosurveillance. Evaluer les influences potentielles que l'art peut jouer dans le processus d'acceptation de la maladie et dans la construction d'une représentation autonome et originelle de celle-ci ;

2) l'équipe : élaborer des savoir-faire pédagogiques intégrant l'art avec les autres membres de l'équipe éducative, dans une perspective d'aider le patient à verbaliser son ressenti, exprimer ses émotions et élaborer des connaissances sur sa maladie et son traitement par des analogies et métaphores artistiques ;

3) l'institution : promouvoir la réalisation au sein de l'hôpital de programmes éducatifs intégrant l'art comme support pédagogique pour favoriser l'apprentissage de l'autosoins et de l'autosurveillance de la part des patients chroniques.

L'acquisition de ces compétences, énoncées ici à un niveau de formulation générale, doit être assurée par la mise en place de formations spécifiques. La participation des soignants à la programmation culturelle et aux initiatives artistiques de l'hôpital peut préparer et sensibiliser les soignants à l'égard de programmes d'éducation thérapeutique intégrant des dimensions artistiques.

\section{Evaluation prévue}

Au-delà de la validation de l'hypothèse théorique, le dispositif d'évaluation devrait permettre de : a) mettre à 
disposition de l'équipe soignante des informations sur l'état psycho-émotionnel des patients ; b) adapter et améliorer le protocole de recherche; c) démontrer l'utilité et la pertinence du programme aux centres décisionnels ${ }^{22}$.

Pour aborder la question de l'évaluation, il est essentiel de répondre aux questions suivantes : qu'est-ce que nous attendons du patient qui a participé à ce programme ? Qu'est-ce qu'il devrait savoir faire après sa participation qu'il ne savait pas faire avant ?

Notre intention est d'aider le patient à être conscient, responsable et actif dans la gestion de sa maladie. La finalité du programme est de développer certaines des capacités nécessaires au patient pour décider de ce qui est le mieux pour lui-même en matière de soins. Deux ordres de capacités sont visés par le programme : les capacités nécessaires à la maîtrise des émotions et les capacités nécessaires à comprendre et appréhender les représentations.

Le patient, après ce programme, devrait être capable de reconnaître, analyser et faire face à ces émotions. Il devrait, par ailleurs, apprendre que la maladie, ainsi que toutes autres expériences, peut avoir un sens différent. Il devrait donc pouvoir s'engager dans une recherche de sens. Ce processus de recherche, en soi et au-delà de la découverte effective, est source de force et de sens. Ce genre de réflexion influence l'image et l'estime que le patient a de lui-même, modifie sa perception de la maladie et du traitement et le rend plus autonome face aux représentations sociales de la maladie.

Pour évaluer si notre hypothèse est valable, nous allons observer si et comment le patient se sent changé. Lobservation peut être réalisée en considérant trois sphères : relation à soi-même, relation à la maladie et au traitement, relation au monde.

Dans chacune de ces sphères, il est possible d'observer les changements par rapport à différents niveaux : a) pour la relation à soi-même : prise de conscience ; (re)découverte de soi ; accroissement de l'estime de soi ; b) pour la relation à la maladie et au traitement : adaptation à la maladie; mise à distance des problèmes liés à la maladie; acceptation de la maladie ; sentiment de bien-être ; c) pour la relation au monde : nouvelle perception du quotidien ; nouvelle perception de la relation avec les proches; nouvelle perception de la relation avec les soignants; identification avec les autres participants et partage.

La finalité de ce programme, comme spécifié plus haut, n'est pas de conduire les participants à des comportements standardisés dans le cadre de la thérapie et de la relation avec les soignants. Par conséquence, ce que nous souhaitons évaluer, c'est plutôt la perception que les patients ont de leur changement à la suite du programme.

Le dispositif d'évaluation est conçu dans l'intention de parvenir aux résultats suivants : a) savoir comment et combien le comportement des participants a évolué, à la suite des quatre rencontres avec les artistes ; b) savoir la place que les participants attribuent aux expériences esthétiques dans la mobilisation des capacités relationnelles et émotionnelles visées par le programme.

Pour estimer le renforcement des capacités relationnelles, nous allons recueillir des informations concernant essentiellement la relation avec les soignants. Nous avons prévu un système de questionnaires croisés : un questionnaire pour les patients et un questionnaire pour les soignants, avec des questions correspondantes afin de comparer les réponses.

Pour estimer le renforcement des capacités d'ordre émotionnel, nous avons prévu des entretiens individuels. Six mois après la fin du programme, le psychologue, avec le médiateur, rencontre chaque participant. Cet entretien est une occasion pour le participant de mettre des mots sur sa perception des expériences vécues pendant les rencontres avec les artistes, sa perception des changements éventuels dans sa sphère psycho-affective et du rôle que l'art a joué dans ces changements. Il s'agit, entre autre, de voir si les participants ont acquis un moyen de gestion de leurs émotions. A titre d'exemple, la fréquentation habituelle de lieux d'art (expositions, théâtres, salles de concerts, etc.) peut devenir un moyen de gestion des émotions. La rencontre avec des œuvres d'art permet de stimuler ou bien d'évacuer ses propres émotions.

\section{Conclusion}

Les potentialités de l'art dans l'éducation thérapeutique se concrétisent dans l'efficacité de l'art comme support pédagogique. Pour réussir le processus d'apprentissage il est essentiel de prendre en compte la sphère émotionnelle ainsi que la sphère cognitive de l'apprenant, ces deux aspects étant indissociables. L'art permet ce double accès. L'expérience esthétique, intégrée dans un programme d'éducation thérapeutique, pourrait favoriser le développement de deux groupes de capacités de la part du patients : 1) pour la gestion des émotions ; 2) pour la prise de conscience d'un décalage entre les représentations personnelles et celles d'autrui, ainsi qu'entre les représentations et la réalité.

L'art mérite d'être exploré et expérimenté comme vecteur d'un changement dans la façon que le patient a de s'appréhender, de se comporter et de se soigner. Le lien entre l'art et l'éducation thérapeutique se réalise au niveau de certains éléments du processus éducatif : réhabilitation du sentiment d'identité et revalorisation de l'image de soi ; acceptation de la maladie ; compréhension du traitement ; 
appréhension des représentations sociales de la maladie ; gestion des émotions. Cette alliance peut déterminer une transformation émotionnelle et cognitive propice au développement des compétences d'autosoins et d'autosurveillance.

La validation de l'hypothèse formulée plus haut ouvre une voie à de nouveaux programmes d'éducation thérapeutique et de formation des professionnels de santé. Ces programmes intégreraient des objectifs pédagogiques de connaissance et de sensibilisation à l'art. Ils permettraient également de découvrir les potentialités de l'art et ses utilisations possibles auprès des patients.

\section{Références}

1. Borillo $M$. Une rencontre symptomatique de la mutation computationnelle de notre temps. Art contemporain et Sciences de la cognition. In : Borillo $M$, Goulette J.P. Cognition et création. Sprimont : Mardaga, 2002:15-31.

2. Pouivet $R$. Conception cognitive de l'art et création artistique. In : Borillo M, Goulette J-P. Cognition et création. Sprimont : Mardaga, 2002:277-86.

3. Umbriaco M, Gosselin L. Emotion, cognition et formation à distance. Revue du Conseil québécois de la formation à distance. $2001 ; 5: 113-22$. [On-line]. Disponible sur : http://cqfd.teluq.uquebec.caldistances/D5_1_g.pdf

4. Goleman D. L'Intelligence émotionnelle. Paris:Laffont, 1997.

5. Lauras-Petit A. De l'émotion à l'intelligence sensible. 1999 [On-line]. Disponible sur : http:/leducation.citesciences. frleducationlapprendrelcommapprends_p5.htm.

6. Lafortune L, Mongeau P. Tenir compte de l'affectivité dans l'apprentissage. Sainte-Foy:Presses de l'Université du Québec, 2002.

7. D'Ivernois J-F, Gagnayre R. Apprendre à éduquer le patient. Paris :Vigot, 1996.

\section{Contributions}

Alessandra Pellecchia a conçu le projet et rédigé le manuscrit. Rémi Gagnayre a assuré l'expertise du travail et participé à la révision du manuscrit.
8. Ginières B, Gagnayre R. La représentation de l'asthme par l'enfant au travers du dessin de la santé : un outil pour la relation éducative. Education du Patient et Enjeux de Santé 2004;3:73-8.

9. Ladrière J. Représentation et connaissance. In : Enciclopedia Universalis; Corpus 19:822-4.

10. Jodelet D. Les représentations sociales. Paris:PUF, 1989.

11. Pellecchia A, Gagnayre R. Art et maladie : perspectives pour l'éducation thérapeutique. Education du Patient et Enjeux de Santé 2004;3:79-84.

12. Bataille P. Un cancer et la vie. Les malades face à la maladie. Paris: Balland, 2003.

13. Rozotte C. L'expérience de la maladie grave comme vecteur d'apprentissage et source de changement per-sonnel. Le cas des malades du cancer. Mémoire de DESS Formations en Santé - Éducation Thérapeutique du Patient. Département de Pédagogie de Sciences de la Santé, Université Paris 13, 2003.

14. Matalon A. Chimiofolies. Forcalquier : HB Editions, 2000.

15. Roy C. Permis de séjour. Paris : Gallimard, 1983. 
16. Verdun S. Vivre avec le cancer. Une infirmière témoigne. Paris : Bayard Éditions, 1992.

17. Sontag S. La maladie comme métaphore. Le SIDA et ses métaphores. Paris: Christian Bourgois Editeur, 1993.

18. Turbiaux M. Le soulier de la morte ou De l'art thérapique. Bulletin de psychologie 2003;468:756-60.

19. Damasio A. Spinoza avait raison. Paris : Odile Jacob, 2003.
20. Haenni C, Anzules C, Assal E, Malavia M, Assal J-P, Golay A. Programme Art et thérapie dans les soins : une nouvelle approche pour le suivi de nos patients. Médecine \& Hygiène 2004;2484:1135-6.

21. WHO Regional Office of Europe, Report of a WHO Working Group. Therapeutic Patient Education, Conti-nuing education programmes for Health Care providers with field of preventing of classic diseases. Copenhagen, 1998.

22. Sudres J-L. Evaluer en Art-thérapie? Entre réalités, illusions et controverses. Le Journal $-F M C-$ Psychiatrie 2002;18:35-9.

Manuscrit reçu le 2 mai 2005 ; commentaires éditoriaux formulés aux auteurs le 28 mars 2006 ; accepté pour publication le 3 avril 2006. 